\title{
Impact of coarse aggregate type and angularity on permanent deformation of asphalt concrete
}

\author{
L. Leon \& R. Charles \\ Department of Civil and Environmental Engineering, \\ University of the West Indies, Trinidad
}

\begin{abstract}
This paper presents a method of evaluating the effect of coarse aggregate type and angularity on hot mix asphalt (HMA) properties and its relationship to the Permanent Deformation resistance. Permanent Deformation is the major pavement distress indicator commonly noted worldwide and is influenced by the properties of the material within the asphalt concrete mix. HMA mixes were prepared using varying coarse aggregate angularity properties such as low, medium and high for two aggregate types. The standardized test methods for classification of aggregate angularity are biased because of the judgement of the test personnel, therefore the study employed the use of the Aggregate Imaging System (AIMS) to evaluate the angularity characteristics. The permanent deformation was measured using the repeated dynamic creep loading test. The research concluded that aggregate type as well as coarse aggregate particle angularity had a significant effect on the Permanent Deformation performance, and also that with an increase in coarse aggregate angularity there was an increase in the resistance of mixes to Permanent Deformation. A comparison between the measured data and predictive data of permanent deformation predictive models showed the limits of existing prediction models. The numerical analysis described the permanent deformation zones and concluded that angularity has an effect on the onset of these zones. Prediction of permanent deformation helps road agencies and by extension economists and engineers determine the best approach for maintenance, rehabilitation, and construction of new road infrastructure.

Keywords: permanent deformation, aggregate angularity, AIMS, asphalt concrete performance, rutting prediction.
\end{abstract}




\section{Introduction}

The nature of construction materials makes it impossible to design a road pavement which does not deteriorate in some way with time and traffic; hence the aim of accurate structural pavement design is to limit the level of distress. An asphaltic concrete mixture is comprised of $90 \%$ aggregate. The other $10 \%$ are the air voids and binder content [1]. These ratios demonstrate the significant impact aggregate can have on controlling rutting. The research focused on the aggregate coarse particle angularity which is defined as the extent to which particle edges are sharp or round [2]. The imaging system which is used to measure the angularity property, defined aggregate angularity as being variations at corners, that is; variations superimposed in the aggregate shape [3] as shown in equation (1).

$$
A I=\sum_{\theta=0}^{355} \frac{\left|R_{\theta}-R_{E E \theta}\right|}{R_{E E \theta}}
$$

where $\mathrm{AI}$ represents the angularity index; $\mathrm{R}_{\theta}$ is the radius of the particle at an angle of $\theta, \mathrm{R}_{\mathrm{EE} \theta}$ is the radius of the equivalent ellipse at an angle of $\theta$. The equivalent ellipse has the same aspect ratio of the particle, but has no angularity (that is smooth with no sharp corners) [3].

Since the 1950s, several methods have been proposed to quantify the form, angularity, and surface texture of aggregate particles [4]. These standardized test methods do not classify all aspects of the aggregate shape. However some researchers have found significant disadvantages in using standardized test in particle classification [4-6] and they can be summarized as follows:

a) The test does not consistently identify angular and cubical aggregates.

b) The test methods have low prediction, precision, and medium practicality.

c) Results are influenced by bulk properties, shape, angularity, and texture.

d) The tests are tedious, labour intensive, time consuming to be used on a daily basis.

A phenomenon that has been examined extensively in the last decade is the use of automated imaging systems for the measurement and classification of angularity. Aggregate Imaging System (AIMS) characterizes the shape of fine and coarse aggregates. It has the ability to analyse the angularity of fine and coarse aggregates [7]. Interesting correlations have been found between aggregate angularity quantified by AIMS and mixture performance [3].

There are many factors that determine the behaviour or performance of a flexible road pavement. One of these performance indicators is the rutting in asphalt-concrete pavements. Permanent Deformation or rutting is caused by the densification and movement of materials under repeated loads, and also might result from lateral plastic flow under the wheel track $[8,9]$. It is also described as a pavement condition indicator defined as a $10 \mathrm{~mm}$ rut or the first appearance of wheel track cracking. This distress occurs primarily by shear failure in HMA [10].

The properties of coarse aggregate materials (physical shape) significantly affect both the strength and stability of asphalt mixes. In an evaluation of the influence of coarse aggregate shape on the strength of asphalt concrete mixtures, it was concluded that cubical particles possessed the best rutting resistance 
compared to the other shapes [11]. This means that coarser and high angular aggregates are expected to perform better than low angular aggregate and by extension the finer gradation mixes. The proper selection of materials is one of the most important tasks in developing an asphalt mixture that shows improved resistance to permanent deformation [12]. Different types of aggregate such as limestone, basalt, dolomite, gravel, granite and traprock have been used for production of asphalt concrete. The high stability has been achieved in using limestone aggregate as compared to basalt aggregate [13].

The prediction of permanent deformation is a challenge of varying complexities, requiring detailed knowledge of materials state, elastic and plastic deformability and viscosity of pavement materials. As depicted in Fig. 1, there are three distinct stages in the relationship between load repetitions and permanent deformation: primary, secondary and tertiary. In the primary zone there is a rapid accumulation of permanent strain; in the secondary zone permanent strain per loading cycle tends to decrease reaching a constant value (linear relationship between loading application and strain); and when permanent strain accumulates rapidly this marks the onset of the tertiary zone.

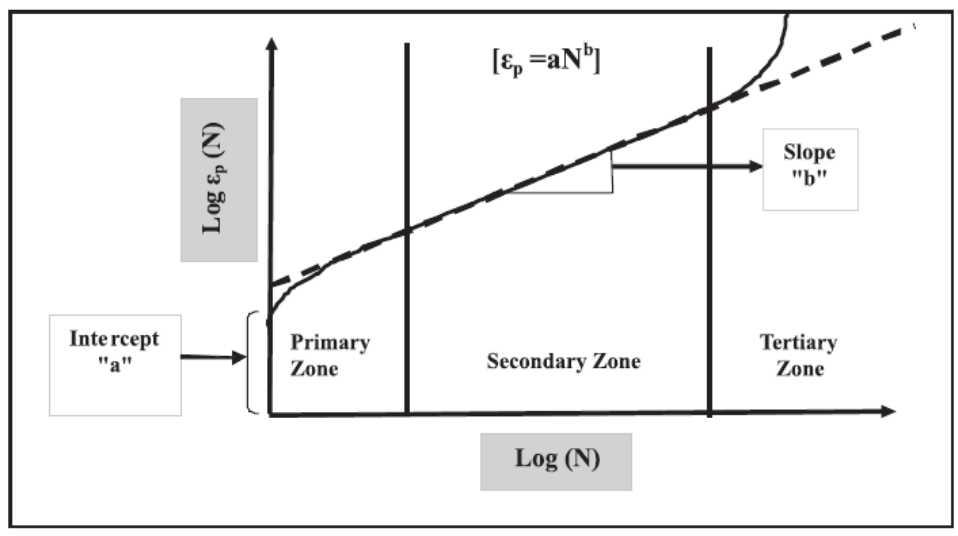

Figure 1: $\quad$ Log-log relationships between load repetition and permanent strain [16].

Within this study the first two stages of Permanent Deformation (Primary and Secondary) were examined. A large number of different permanent deformation models are available, such as:

MEPDG:

NCHRP 1-40B:

and VESYS:

$$
\frac{\varepsilon_{p}}{\varepsilon_{r}}=k_{z} 10^{k_{r 1}} T^{k_{r 3}} N^{k_{r 2}}
$$


However, when these models are given the same input data, they produce very different output (predictions). It is important that these models be easily adjustable in accordance with the available historical data and the engineer's knowledge of local materials and environmental effects. The available permanent deformation prediction models have several limitations in that most of them involve large simplifications (e.g. in material behaviour), some of them contain input factors that are difficult to quantify and most are not comprehensive enough (fails to consider all influencing factors). Regarding rutting prediction models found in the Mechanistic Empirical Pavement Design Guide (MEPDG) and by extension National Cooperative Highway Research Program (NCHRP) 1-40B, have specific parameters and do not need to run laboratory testing. In one instance it should be noted that such models which do not necessitate laboratory testing carries the inherent advantage of being simple and lest time-consuming to implement. However, it is equally important to note that failure to use laboratory characterizations of HMA mixes has the potential to result in inaccurate rutting predictions.

This research study provides evidence of the variability of the predictions between existing models, as well as a comparison between existing models predictive data and this study data (with the adjustment of aggregate angularity property). In spite of an enormous effort that has been made in the pavement engineering field, it still is not possible to make accurate and precise predictions of pavement life. Preservation of road infrastructure assets require a systematic approach such as performance modelling to help in the development of tactical and strategic plans. Pavement performance predictive models allow the forward prediction of future conditions based on the present conditions under a defined range of scenarios [18].

\section{Materials and testing methods}

\subsection{Material}

Commonly used construction materials Natural Quartzite and Crushed Limestone were the two types of aggregate used in this study. The aggregates produced respective gradations as shown in Table 1. The mixes were dense graded mixes; and they were classified under three (3) categories which were governed by coarse aggregate angularity within the mix (low, medium and high angularity). Trinidad Lake Asphalt (TLA) was the study binder. Aggregate abrasion tests evaluated the wear potential of each type of aggregate.

\subsection{AIMS imaging system test}

The Aggregate Imaging System (AIMS) was used for imaging analysis to characterize the angularity characteristics of coarse aggregate particles. The test samples were prepared with varying coarse aggregate angularity properties such as low, medium and high. The classification properties of both quartzite and limestone coarse aggregate particles for the mixes, and also the identification of the angularity designations are shown in Table 1. 
Table 1: The properties of the coarse aggregate materials.

\begin{tabular}{|c|c|c|c|c|c|}
\hline $\begin{array}{c}\text { Aggregate } \\
\text { Type }\end{array}$ & $\begin{array}{c}\text { Abrasion } \\
\text { (wear \%) }\end{array}$ & $\begin{array}{c}\text { Coarse to } \\
\text { Fine Ratio }\end{array}$ & $\begin{array}{c}\text { Angularity } \\
\text { ID }\end{array}$ & $\begin{array}{c}\text { AIMS Aggregate } \\
\text { Angularity Number }\end{array}$ & $\begin{array}{c}\text { MIX } \\
\text { ID }\end{array}$ \\
\hline $\begin{array}{c}\text { Quartzite } \\
(\mathrm{Q})\end{array}$ & $44 \%$ & $46 \%$ to $54 \%$ & Low & $<2999$ & QL \\
\cline { 4 - 6 } & & & High & $>6000$ & QH \\
\hline \multirow{2}{*}{$\begin{array}{c}\text { Limestone } \\
(\mathrm{L})\end{array}$} & $30.5 \%$ & $48 \%$ to 52\% & Medium & $3000-5999$ & LM \\
\cline { 4 - 6 } & & & High & $>6000$ & LH \\
\hline
\end{tabular}

\subsection{Mix design and performance test}

The mix design was conducted using the current and commonly used method of mix design which is the Marshall Mix design procedure. All mixes met the Programme for Upgrading Roads Efficiency (PURE) agency standards of acceptable limits of mix properties. The blend of aggregates for both material types used in the various mixes had no statistically significant difference between the two gradations $(\mathrm{p}=0.929>0.05$ mean; $\mathrm{p}=0.937>0.05$ standard deviation $)$; therefore the research aim of aggregate type effect was accurately examined.

Permanent Deformation resistance of the mixes were evaluated using the repeated loading dynamic creep test. The applied test stress was $200 \mathrm{kPa}$. The testing cycle stops after 3,000 loading applications. The equilibrium test temperature was $35^{\circ} \mathrm{C}$.

\section{Results and analysis}

\subsection{Aggregate angularity on permanent deformation}

Fig. 2 shows that all the mixes exhibited the theoretical behaviour mentioned in previous studies [15]. However the tertiary stage was not evident in the measured or predictive results of this study. The results obtained from the dynamic creep test also demonstrated similar results for all mixes with different aggregate type and angularity. Mixtures with aggregates that have low resistance to wear (quartzite) had very low resistance to permanent deformation as compared to limestone material which has a high wear resistance. Even if the mixes were of different categories of angularity (low, medium, high) the results revealed that the type of aggregate significantly affects the resistance to permanent deformation. 

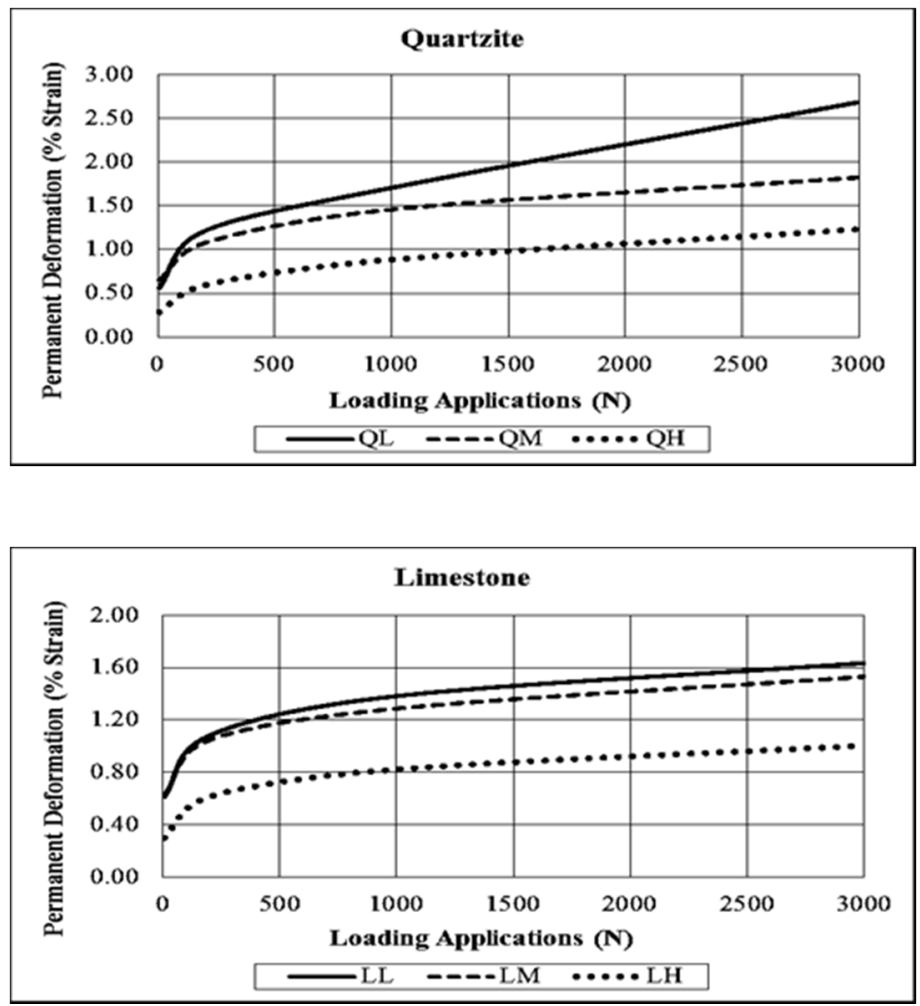

Figure 2: Coarse aggregate type and angularity on permanent deformation.

\subsection{Permanent deformation prediction versus measured data}

The results in Fig. 3 shows that the high angular aggregates have higher resistance to rutting, unlike medium and low which have almost the same measured values as each other. As the angularity of coarse aggregate changes to be more angular, the internal resistance increases and the HMA mix improves its capacity to carry traffic load. The high angularity particles possess the highest deformation resistance, followed by medium and low angular particles. It thus appears that a HMA mix can be made more stable and resistant to deformation by specifying the coarse aggregate angularity. Fig. 3 also shows a comparison of the results of two permanent deformation prediction models when compared with the actual measured laboratory results.

The NCHRP model has a model input for angularity ( $F_{\text {index }}$ and $\left.\mathrm{C}_{\text {index }}\right)$, however, the model still underestimates the percentage of deformation in an HMA mix. The MEPDG however overestimates deformation. This probably means that these models lack a more rigorous variable which accounts for the potential aggregate particle angularity. 

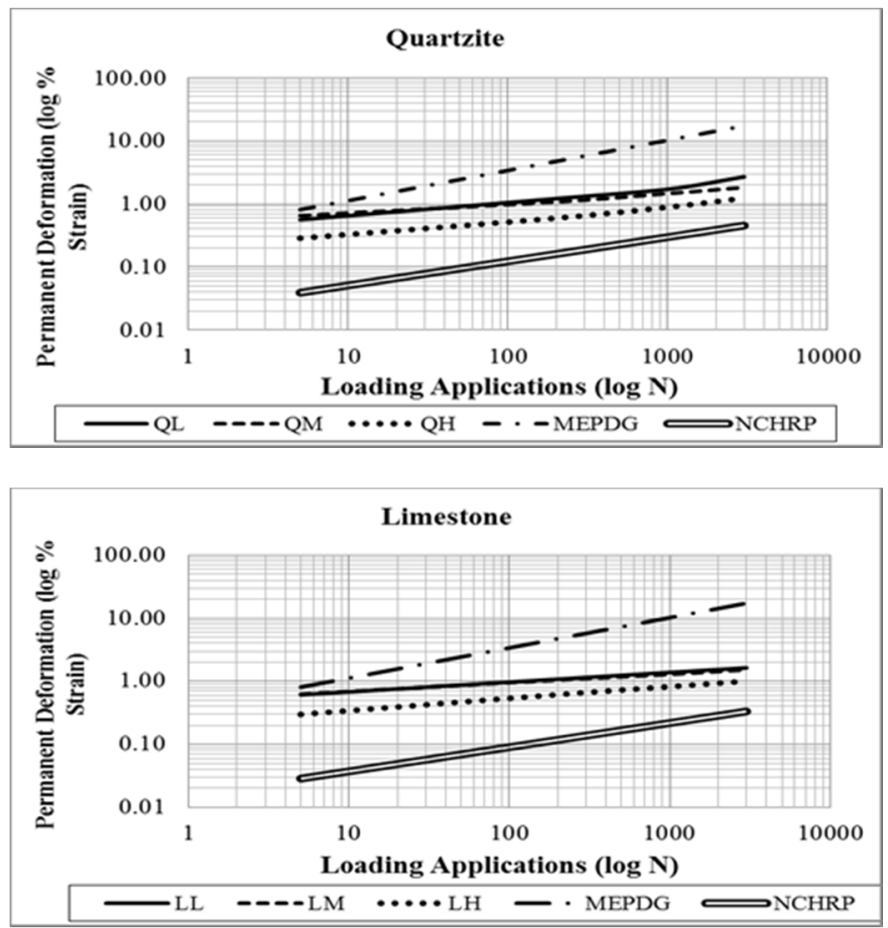

Figure 3: Coarse aggregate type and angularity on permanent deformation.

\section{Permanent deformation zones}

As shown in Fig. 1, the various zones of permanent deformation versus loading application can be modelled. Equations (5) to (7) give the mathematical explanation of the permanent deformation zones as previously mentioned.

Primary Zone:

$$
\begin{aligned}
& \varepsilon_{p}=a N^{b} \\
& \varepsilon_{p}=d N+c \\
& \varepsilon_{p}=f \exp ^{g N}
\end{aligned}
$$

Each equation's parameters ( $\mathrm{a}, \mathrm{b}, \mathrm{c}, \mathrm{d}, \mathrm{f}$, and $\mathrm{g}$ ) can be determined by regression analysis once the strain and load application cycles are known. To determine the start of the secondary and tertiary zone the use of numerical analysis can be employed.

An iterative method is commonly described as a mathematical procedure that produces a sequence of approximate solutions which are expected to converge to the true or exact solution of a particular problem. There are a number of iterative methods, such methods include, the Jacobi method, the Gauss-Seidel method and the Secant method. These methods have been tried and used successfully in various numerical techniques and mathematical problem cases. 
This paper, however, employs the Newton-Raphson method, which is an iterative numerical method for finding the solution or roots of equations arising from differential equations. The Newton-Raphson method is based on the idea of linear approximation where the function must be differentiable. The NewtonRaphson technique is given by the formula:

$$
x_{i+1}=x_{i}-\frac{f\left(x_{i}\right)}{f^{\prime}\left(x_{i}\right)}
$$

where, $f(x)$ is the function to be solved and $f^{\prime}(x)$, is the derivative of the function, $x_{i}$, is the initial or guess value and $x_{i+1}$, represents the calculated or new value. As mentioned previously the zones of permanent deformation can be represented mathematically, therefore using the combined equations (5) and (6) for secondary zone initial transition point and equations (6) and (7) for tertiary zone, a concise explanation of the Newton-Raphson method used in this work is described as follows:

$$
\begin{gathered}
N_{i+1, \mathrm{sec}}=N_{i}-\left[\frac{a N^{b}-d N-c}{a b N^{b-1}-d}\right] \\
N_{i+1, \text { ter }}=N_{i}-\left[\frac{d N+c-f \exp ^{g N}}{d-f g \exp ^{g N}}\right]
\end{gathered}
$$

where, $N$ is the variable and regression coefficients are represented by a, b, c, d, f and $g$. To find the approximate root of $f(x)$, an initial solution of $f(x)$ was assumed, in this case, $N=5$. Then it was necessary to find out the amount of accuracy required in the approximations. That is the tolerance error. This value was taken to be $1 \times 10^{-7}$.

The formula of the Newton-Raphson method stated in equation (9) above was then solved in an iterative numerical manner. From this relationship, the value of $N_{i+1}$, the difference between the current and previous values of $N$ was estimated.

At the end of each iterative step the difference was compared to the accuracy value. If the difference is less than the accuracy value, then iteration is stopped and the current value of $N$ is considered as the approximate root of the solution. If, however, the difference remained larger than the accuracy value even after a large number of iteration, then the solution will be said to be non-converging.

The maximum loading applications within the research was 3000 cycles. The samples did not reach the tertiary zone; therefore the results presented were of the algorithm for obtaining the transition point between primary and the secondary stage which is stated by equation (9).

The result in Table 2 shows that as coarse aggregate angularity increases so does the onset of the secondary stage. It also indicates a lower permanent deformation strain estimate for high angularity. Various models such as the VESYS rutting prediction model, use the strain estimate at the $200^{\text {th }}$ cycle to predict deformation depth with a pavement structure. However from the research algorithm the VESYS assumption can be affected by the type or abrasion property of the aggregate. Limestone has a higher resistance to abrasion compared to 
quartzite. Limestone $200^{\text {th }}$ loading application cycle occurred in the primary zone while for quartzite it occurred in the secondary zone.

Table 2: Transition points between primary and secondary zone.

\begin{tabular}{|c|c|c|c|c|c|}
\hline \multirow{2}{*}{$\begin{array}{c}\text { Aggregate } \\
\text { Type }\end{array}$} & $\begin{array}{c}\text { Angularity } \\
\#\end{array}$ & $\begin{array}{c}\text { No. of } \\
\text { Iterations }\end{array}$ & $\begin{array}{c}\text { Load } \\
\text { Application } \\
\text { N }_{\text {sec }}\end{array}$ & $\begin{array}{c}\text { Permanent } \\
\text { Deformatio } \\
\text { n Strain, } \\
\varepsilon_{\mathrm{p}, \mathrm{sec}}\end{array}$ & $\begin{array}{c}\text { Permanent } \\
\text { Deformation } \\
\text { Strain @ 200 } \\
\text { cycle, } \varepsilon_{\mathrm{p}, 200 t h}\end{array}$ \\
\hline & 2019 (low) & 5 & 105.6 & 1.0818 & 1.1397 \\
\cline { 2 - 6 } & 6176 (high) & 13 & 113.4 & 0.5371 & 0.5607 \\
\hline \multirow{2}{*}{ Limestone } & 2770 (low) & 7 & 327.1 & 1.1712 & 1.0853 \\
\cline { 2 - 6 } & 6117 (high) & 10 & 477.7 & 0.5417 & 0.4337 \\
\hline
\end{tabular}

\section{Summary and conclusions}

If an asphalt mixture deforms (ruts), it is normally because the mixture has insufficient shear strength to support the stresses to which it is subjected. Aggregates are responsible for minimizing shear failure within an asphalt concrete mix. From the experimental study conducted it can be concluded that the aggregate resistance to degradation (abrasion wearing) is significantly influenced by the aggregate type and by extension its morphological properties. HMA mix density and stability properties can be vastly affected by the aggregate type abrasion wear potential. The higher the abrasion wear resistance, the higher the mix density and greater stability properties when used within a mix.

The AIMS imaging system was shown to be a useful tool for quantifying the angularity characteristics of coarse aggregate. It quantifies the angularity as well as other shape properties for each individual particle within. The analysis of the angularity data is not subjected to human error which leads to more accurate results. For any given type of aggregate, an increase in the coarse aggregate particle angularity in a mix decreases its susceptibility to permanent deformation, while increasing stability potential.

The proposed algorithm for the estimates of the onset of secondary zone was used for different aggregate type with varying levels of coarse aggregate angularity. The existing predictive models did not accurately predict deformation of the mixes because the material properties input were subjected to a user bias test. The research procedure validates that the transition points of permanent deformation zone can be estimated using mathematical models that describe each zones.

The accuracy in the prediction of HMA mixes to permanent deformation can be obtained if prediction models take into account a more accurate or an additional variable for the aggregate particle angularity property. 


\section{References}

[1] Janoo V. C., \& Korhonen, C., Performance Testing of Hot Mix Asphalt Aggregates, US Army Corps of Engineers, Cold Regions Research \& Engineering Laboratory, 1999.

[2] Wadell, H., Volume, Shape and Roundness of Rock Particles, Journal of Geology, vol. 40, pp. 443-451, 1932.

[3] Masad, E. A., Aggregate Imaging System (AIMS): Basics and Application, Texas Department of Transportation, 2004.

[4] Lee, C. J., West, T. R. \& White, T. D., Effect of Fine Aggregate Angularity on Asphalt Mixture Performance, Joint Transportation Research Program, 1999.

[5] Rao, C., Stefanski, J. A. \& Tutumleur, E., Video Image Analysis of Aggregates, Civil and Engineering Studies, Transportation Engineering Series No. 111, Illinois Cooperative Highway and Transportation Series No. 278, 2000.

[6] Button, J. W., Chowdhury, A., Jahn, D. \& Kohale, V., Evaluation of Superpave Fine Aggregate Angularity Specification, International Center for Aggregate Research (ICAR), Texas Transportation Institute, 2001.

[7] Chandan, C., Fletcher, T., Masad, E. \& Sivakumar, K., Aggregate Imaging System (Aims) For Characterizing The Shape of Fine and Coarse Aggregates, 82nd Annual Transportation Research Board for Presentation and Publication, 2002.

[8] NCAT, Hot Mix Asphalt Materials, Mixture Design and Construction, 2nd ed., Maryland: National Center of Asphalt Technology, NAPA Research and Education Foundation, 2000.

[9] Dessouky, S., Little, D. \& Masad, E., Mechanistic Model to Predict the Impact of the Aggregate Mixture on the Permanent Deformation of Asphalt Mixtures, Texas Transportation Institute, 2005.

[10] Brown, S. F., \& Brunton, J. M., An Introduction to the Analytical Design of Bituminous Pavements 3rd Edition, UK: University of Nottingham, 1987.

[11] Chang, M. K., Chen, J. S., \& Lin, K. Y., Influence of Coarse Aggregate Shape on the Strength of Asphalt Concrete Mixtures, Journal of the Eastern Asia Society for Transportation Studies, pp. 1062-1075, 2005.

[12] Button, J. W., \& Yeggoni, M., Influence of Coarse Aggregate Shape and Surface Texture on Rutting of Hot Mix Asphalt Concrete, 1994.

[13] M. A. Ahmed and A. Mohammed, "Impact of Aggregate Gradation and Type on Hot Mix Asphalt Rutting in Egypt", International Journal of Engineering Research and Application (IJERA), vol. 3, pp. 2249-2258, 2013.

[14] J. E. Haddock, E. Rismantojo and T. D. White, "Aggregates Test for Hot Mix Asphalt Mixture Used in Pavements", National Cooperative Highway Research Program, NCHRP, 2006.

[15] O. Ali, "Evaluation of the Mechanistic Empirical Pavement Design Guide", Institute of research in Construction, National Research Council, Canada, 2005. 
[16] I. Hafeez, "Impact of Hot Mix Asphalt Properties on its Permanent Deformation Behaviour", 2009.

[17] A. A. Molenaar, "Pavement Performance, Evaluation and Rehabilitation Design", in Proceedings of MAIREPAV 03, Guimaraes, Portugal, 2003.

[18] E. Fernando, T. Scullion and F. Zhou, "A Review of Performance Models and Test Procedures with Recommendations for Use in the Texas M-E Design Program", Texas Transportation Institute, The Texas A\&M University System, 2008. 